\title{
SOLVING FUZZY TRANSPORTATION PROBLEM USING RANKING OF OCTAGONAL FUZZY NUMBERS
}

\author{
P. Malini ${ }^{1}$, M. Ananthanarayanan ${ }^{2}$ \\ ${ }^{1}$ Department of Mathematics \\ Jeppiaar Engineering College \\ Chennai, 119, INDIA \\ ${ }^{2}$ Department of Mathematics \\ A.M. Jain College \\ Chennai, 114, INDIA
}

\begin{abstract}
Octagonal fuzzy numbers, is applied to a fuzzy valued transportation problem. Using this ranking method we convert any fuzzy transportation problem (cost, demand and supply appearing as octagonal fuzzy numbers) to a crisp valued transportation problem which then can be solved using the MODI method. The proposed method serves as an efficient method in ranking Octagonal fuzzy numbers which is illustrated through a numerical example.
\end{abstract}

AMS Subject Classification: 90BXX

Key Words: Octagonal Fuzzy numbers, Fuzzy Transportation Problem.

\section{Introduction}

The transportation problem was originally introduced and developed by Hitch Cock in 1941, in which the parameters like transportation cost, demand and supply are crisp values. But in the present world the transportation parameters may be uncertain due to many uncontrolled factors. The transportation problem is a special case of Linear programming problem,

Received: $\quad$ March 26, 2016

Revised: $\quad$ August 23, 2016

Published: November 4, 2016

${ }^{\S}$ Correspondence author (c) 2016 Academic Publications, Ltd. url: www.acadpubl.eu 
which permit us to determine the optimum shipping patterns between origins and destinations. The solution of the problem will empower us to determine the number of units to be transported from a particular origin to a particular destination so that the cost obtained is minimum or the time taken is minimum or the profit obtained is maximum.

Let $a_{i}$ be the number of units of a product available at origin $i$ and $b_{j}$ be the number of units of the product required at destination $j$. Let $C_{i j}$ be the cost of transporting one unit from origin $i$ to destination $j$ and let $x_{i j}$ be the amount of quantity carried or shipped from origin $i$ to destination $j$. A fuzzy transportation problem is a transportation problem in which the transportation expenditures, supply and demand quantities are fuzzy quantities. Michael [9] has proposed an algorithm for elucidating transportation problems with fuzzy constraints and has discussed the relationship between the algebraic structure of the fuzzy optimum solution of the deterministic problem and its fuzzy equivalent. Nagoor Gani and Abdul Razack [10] procured a fuzzy solution for a two stage cost minimizing fuzzy transportation problem. Fuzzy numbers must be ranked before a decision is taken by a decision maker. Ranking normal fuzzy numbers were first introduced by Jain [5] for decision making in fuzzy situations. The fuzzy set theory has been applied in many fields such as Management, Engineering etc. Pandian and Natarajan [11] proposed a algorithm namely fuzzy Zero point method for finding optimal solution of a fuzzy transportation problem with trapezoidal fuzzy numbers. Few of these ranking methods have been reviewed and compared by Bortolan and Degani [2]. Presently Chen and Hwang reviewed the existing methods for ranking fuzzy numbers and each approach has drawbacks in some aspects [4]. Ranking fuzzy numbers has attracted research attention due to their vast applications in the theory of fuzzy decision making, risk analysis, data analysis, optimization, etc. Several ranking methods have been proposed by many authors since $1980[1,3,6,7,8,12]$.

In this paper a new ranking method is introduced on octagonal fuzzy numbers. By using this ranking the fuzzy transportation problem is converted to a crisp valued problem, which could be solved using VAM method for initial solution and MODI method for optimal solution. The optimal solution could be got either as a fuzzy number or as a crisp number.

The paper is organized as follows : In Section 2, the notion of fuzzy number and octagonal fuzzy numbers are recalled. In Section 3, the ranking method is proposed for octagonal fuzzy numbers. In Section 4, a numerical example (FTP) is solved to illustrate the proposed ranking method. Finally the paper ends with a conclusion. 


\section{Preliminaries}

In this section we define some basic definitions which will be applied in this paper.

Definition 2.1. If $x$ is a collection of objects denoted generally by $X$, then a fuzzy set $A$ in $X$ is explained as a set of ordered pairs $A=\left\{\left(x, \mu_{A}(x) / x \in X\right\}\right.$ where $\mu_{A}(x)$ is termed as the membership function for the fuzzy set $A$. The membership function maps each element of $X$ to a membership value between 0 and 1.

Definition 2.2. A fuzzy set $A$ is defined on universal set of real numbers is said to be a generalized fuzzy number if its membership function has the following attributes:

(i) $\mu_{A}(x): R \rightarrow[0,1]$ is continuous;

(ii) $\mu_{A}(x)=0$ for all $x \in A(-\infty, a] \cup[d, \infty)$;

(iii) $\mu_{A}(x)$ is strictly increasing on $[a, b]$ and strictly decreasing on $[c, d]$;

(iv) $\mu_{A}(x)=w$ for all $x \in[b, c]$, where $0<w \leq 1$

Definition 2.3. A generalized fuzzy number $A=(a, b, c, d, w)$ is said to be a generalized trapezoidal fuzzy number if its membership function is given by

$$
\mu_{A}(x)= \begin{cases}\frac{w(x-a)}{b-a}, & a \leq x \leq b \\ w, & b \leq x \leq c \\ \frac{w(x-a)}{d-c}, & c \leq x \leq d \\ 0, & \text { otherwise }\end{cases}
$$

If $w=1$, then $A=(a, b, c, d ; 1)$ is a normalized trapezoidal fuzzy number and $A$ is a generalized or non normal trapezoidal fuzzy number if $0<w<1$

As a particular case if $b=c$, the trapezoidal fuzzy number reduces to a triangular fuzzy number given by $A=(a, b, c ; w)$

Definition 2.4. A fuzzy number $A$ is a normal octagonal fuzzy number expressed by $\left(a_{1}, a_{2}, a_{3}, a_{4}, a_{5}, a_{6}, a_{7}, a_{8}\right)$ where $a_{1}, a_{2}, a_{3}, a_{4}, a_{5}, a_{6}, a_{7}, a_{8}$ are 
real numbers and its membership function $\mu_{A}(x)$ is given below

$$
\mu_{A}(x)= \begin{cases}0, & \text { for } x<a_{1} \\ \frac{k\left(x-a_{1}\right)}{\left(a_{2}-a_{1}\right)}, & \text { for } a_{1} \leq x \leq a_{2} \\ k, & \text { for } a_{2} \leq x \leq a_{3} \\ k+(1-k) \frac{\left(x-a_{3}\right)}{\left(a_{4}-a_{3}\right)}, & \text { for } a_{3} \leq x \leq a_{4} \\ 1, & \text { for } a_{4} \leq x \leq a_{5} \\ k+(1-k) \frac{\left(a_{6}-x\right)}{\left(a_{6}-a_{5}\right)}, & \text { for } a_{5} \leq x \leq a_{6} \\ k, & \text { for } a_{6} \leq x \leq a_{7} \\ k \frac{\left(a_{8}-x\right)}{\left(a_{8}-a_{7}\right)}, & \text { for } a_{7} \leq x \leq a_{8} \\ 0, & \text { for } x>a_{8}\end{cases}
$$

where $0<k<1$

Remark 2.5. If $k=0$, the octagonal fuzzy number reduces to the trapezoidal fuzzy number $\left(a_{3}, a_{4}, a_{5}, a_{6}\right)$ and if $k=1$, it reduces to the trapezoidal fuzzy number $\left(a_{1}, a_{4}, a_{5}, a_{8}\right)$.

Definition 2.6. A fuzzy number $A$ is said to be a generalized octagonal fuzzy number denoted by $A=\left(a_{1}, a_{2}, a_{3}, a_{4}, a_{5}, a_{6}, a_{7}, a_{8} ; k, w\right)$ where $a_{1}, a_{2}, a_{3}$, $a_{4}, a_{5}, a_{6}, a_{7}, a_{8}$ are real numbers and its membership function $\mu_{A}$ is given by

$$
\mu_{A}(x)= \begin{cases}0, & \text { for } x<a_{1} \\ \frac{k\left(x-a_{1}\right)}{\left(a_{2}-a_{1}\right)}, & \text { for } a_{1} \leq x \leq a_{2} \\ k, & \text { for } a_{2} \leq x \leq a_{3} \\ k+(w-k) \frac{\left(x-a_{3}\right)}{\left(a_{4}-a_{3}\right)}, & \text { for } a_{3} \leq x \leq a_{4} \\ w, & \text { for } a_{4} \leq x \leq a_{5} \\ k+(w-k) \frac{\left(a_{6}-x\right)}{\left(a_{6}-a_{5}\right)}, & \text { for } a_{5} \leq x \leq a_{6} \\ k, & \text { for } a_{6} \leq x \leq a_{7} \\ k, \frac{\left(a_{8}-x\right)}{\left(a_{8}-a_{7}\right)}, & \text { for } a_{7} \leq x \leq a_{8} \\ 0, & \text { for } x>a_{8}\end{cases}
$$

\section{Ranking of Octagonal Fuzzy Numbers}

A number of approaches have been proposed for the ranking of fuzzy numbers. Ranking methods map fuzzy number directly into the real line. That is $M$ : $F \rightarrow R$ which associate every fuzzy number with a real number. 


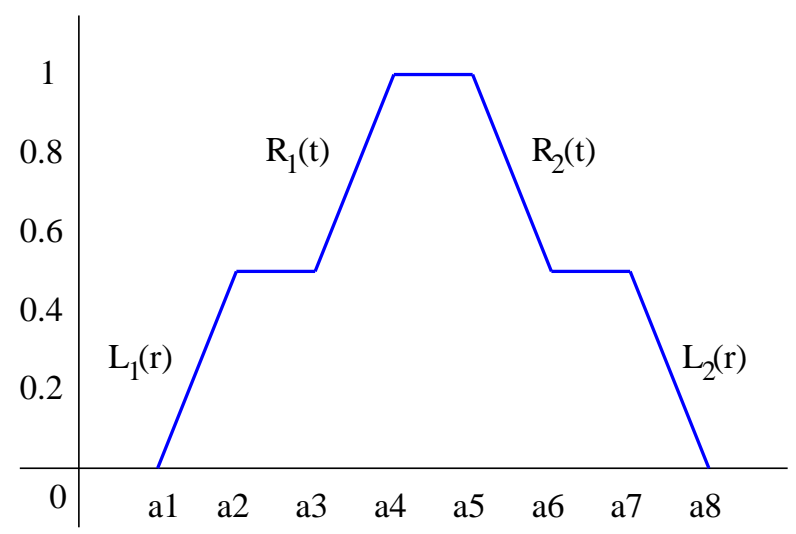

Figure 1: Graphical representation of the octagonal fuzzy number $A=$ $\left(a_{1}, a_{2}, a_{3}, a_{4}, a_{5}, a_{6}, a_{7}, a_{8} ; 0.5,1\right)$

Let $L_{1}^{-1}(r), L_{2}^{-1}(r), R_{1}^{-1}(t)$ and $R_{2}^{-1}(t)$ are the inverse function of $L_{1}(r)$, $L_{2}(r), R_{1}(t)$ and $R_{2}(t)$ respectively.

Let $A$ be a generalized octagonal fuzzy number. The ranking of $A$ is calculated as follows:

$$
\begin{aligned}
R(A)= & \frac{\frac{1}{4} \int_{0}^{k} x L_{1}^{-1}(x) d x}{\int_{0}^{k} x d x}+\frac{\frac{1}{4} \int_{k}^{w} x L_{2}^{-1}(x) d x}{\int_{k}^{w} x d x}+\frac{\frac{1}{4} \int_{w}^{k} x R_{1}^{-1}(x) d x}{\int_{w}^{k} x d x} \\
+ & \frac{\frac{1}{4} \int_{k}^{0} x R_{2}^{-1}(x) d x}{\int_{k}^{0} x d x} \\
= & \frac{1}{2}\left[\frac{a_{1}+a_{3}+a_{6}+a_{8}}{2}+\frac{a_{2}-a_{1}+a_{7}-a_{8}}{3}\right. \\
& \left.+\frac{a_{4}-a_{3}}{w-k}\left(\frac{w^{2}+w k+k^{2}}{3(w+k)}-\frac{k}{2}\right)+\frac{a_{6}-a_{5}}{w-k}\left(\frac{k}{2}-\frac{k^{2}+w^{2}+w k}{3(w+k)}\right)\right] .
\end{aligned}
$$

If $w=1, k=\frac{1}{2}$, then

$$
R(A)=\frac{3 a_{1}+6 a_{2}+4 a_{3}+5 a_{4}+5 a_{5}+4 a_{6}+6 a_{7}+3 a_{8}}{36} .
$$

Remark 3.1. If $A_{w}$ and $B_{w}$ are two octagonal fuzzy numbers and $w \in$ $(0,1)$ then we have:

1. $A_{w} \leq B_{w} \Leftrightarrow R_{w}(A) \leq R_{w}(B) ;$

2. $A_{w}=B_{w} \Leftrightarrow R_{w}(A)=R_{w}(B)$;

3. $A_{w} \geq B_{w} \Leftrightarrow R_{w}(A) \geq R_{w}(B)$. 


\section{Numerical Example}

We shall present a solution to fuzzy transportation problem involving shipping cost, customer demand and availability of products using octagonal fuzzy numbers. Consider the following fuzzy transportation problem.

\begin{tabular}{|c|c|c|c|c|c|}
\hline & \multicolumn{4}{|c|}{ DESTINATION } & SUPPLY \\
\hline \multirow[t]{3}{*}{ SOURCE } & $\begin{array}{l}(-1,0,1, \\
2,3,4,5, \\
6)\end{array}$ & $\begin{array}{l}(0,1,2, \\
3,4,5,6, \\
7)\end{array}$ & $\begin{array}{ll}(8, & 9, \\
11, & 12, \\
13, & 14, \\
15) & \end{array}$ & $\begin{array}{lll}(4, & 5, & 6, \\
7, & 8, & 9, \\
10, & 11)\end{array}$ & $\begin{array}{lll}(1, & 3, & 5, \\
6, & 7, & 8, \\
10, & 12)\end{array}$ \\
\hline & $\begin{array}{l}(-2,-1,0 \\
1,2,3,4, \\
5)\end{array}$ & $\begin{array}{l}(-3,-2,- \\
1,0,1,2, \\
3,4)\end{array}$ & $\begin{array}{l}(2,4,5 \\
6,7,8,9 \\
11)\end{array}$ & $\begin{array}{l}(-3,-1,0 \\
1,2,4,5, \\
6)\end{array}$ & $\begin{array}{l}(-2,-1,0, \\
1,2,3,4, \\
5)\end{array}$ \\
\hline & $\begin{array}{l}(2,3,4, \\
5,6,7,8, \\
9)\end{array}$ & $\begin{array}{l}(3,6,7, \\
8,9,10, \\
12,13)\end{array}$ & $\begin{array}{l}(11, \quad 12, \\
14, \quad 15, \\
16, \\
18,21)\end{array}$ & $\begin{array}{l}(5,6,8, \\
9,10,11, \\
12,15)\end{array}$ & $\begin{array}{lr}5, & 6, \\
10, & 12, \\
13, & 15, \\
17) & \end{array}$ \\
\hline DEMAND & 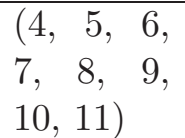 & $\begin{array}{l}(1,2,3, \\
5,6,7,8, \\
10)\end{array}$ & $\begin{array}{l}(0,1,2, \\
3,4,5,6, \\
7)\end{array}$ & $\begin{array}{l}(-1,0,1, \\
2,3,4,5, \\
6)\end{array}$ & \\
\hline
\end{tabular}

Step 1: Now by using the ranking technique, we convert the given fuzzy problem in to a crisp value problem. The problem is done by taking the value of $k$ as 0.5 . The FTP is

\begin{tabular}{|c|c|c|c|}
\hline 2.5 & 3.5 & 11.5 & 7.5 \\
\hline 1.5 & 0.5 & 6.5 & 1.78 \\
\hline 5.5 & 8.585 & 15.415 & 9.415 \\
\hline 7.5 & 5.22 & 3.5 & 2.5
\end{tabular}

Step 2: Using VAM procedure we obtain the initial solution as

\begin{tabular}{|c|c|c|c|}
\hline 1.28 & 5.22 & & \\
\hline & & & 1.5 \\
\hline 6.22 & & 3.5 & 1 \\
\hline
\end{tabular}

The minimum cost is 121.7175 Which is not an optimal solution

Step 3: Hence by using the MODI method we shall get the optimal solution as

\begin{tabular}{|l|l|l|l|}
\hline & 5.22 & 1.28 & \\
\hline & & & 1.5 \\
\hline 7.5 & & 0.72 & 2.5 \\
\hline
\end{tabular}


The crisp solution to the transportation problem is Rs 118.6263

Remark 4.1. If we convert the octagonal fuzzy transportation problem in to trapezoidal fuzzy transportation problem and it is solved as in [11] we get the crisp value of the optimum fuzzy transportation cost is $\mathrm{z}=\operatorname{Rs} 140.83$

\section{Conclusion}

In this paper a simple method of solving fuzzy transportation were introduced by using ranking of fuzzy numbers. We infer the results found were satisfactory and this has been proved with illustrative example. The proposed ranking procedure can be applied in various decision making problems. It is more efficient and simple when compared to the earlier methods. Also it gives us the optimum cost which is very much lower when it is solved using trapezoidal fuzzy numbers.

\section{References}

[1] S. Abbasbandy and T. Hajjari, A new approach for ranking of trapezoidal fuzzy numbers, Computers and Mathematics with Applications, 57 (2009), 413-419.

[2] G. Bortolan and R. Degani, A review of some methods for ranking fuzzy subsets, Fuzzy Sets and Systems, 15 (1985), 1-19.

[3] C.H. Cheng, A new approach for ranking fuzzy numbers by distance method, Fuzzy Sets and Systems, 95 (1998), 307-317.

[4] S.J. Chen and C.L. Hwang, Fuzzy multiple attribute decision making, (1992), Springer, Berlin.

[5] R. Jain, Decision making in the presence of fuzzy variables, IEEE Transactions on systems, Man and Cybernetics, 6 (1976), 698-703.

[6] A. Kaufmann and M.M. Gupta, Introduction to Fuzzy Arithmetics, Theory and Applications, Van Nostrand Reinhold, Newyork (1985).

[7] L.W. Lee and S.M. Chen, Fuzzy risk analysis based on fuzzy numbers with different shapes and different deviations, Experts Systems with Applications, 34 (2008), 27632771.

[8] P. Malini and M. Ananthanarayanan, Solving fuzzy assignment using ranking of generalized trapezoidal fuzzy numbers, Indian Journal of Science and Technology, 9(20) (2016), 1-4.

[9] Micheal OhEigeartaigh, A fuzzy transportation algorithm, Fuzzy Sets and System, 8 (1982), 235-243.

[10] A. Nagoor Gani and K. Abdul Razack, Two stage fuzzy transportation problem, Journal of Physical Science, 10 (2006), 63-69. 
[11] P. Pandian and G. Natarajan, A new algorithm for finding a fuzzy optimal solution for fuzzy transportation, Applied Mathematical Sciences, 4 (2010), 79-90.

[12] P. Phani Bushan Rao and N. Ravishankar, Ranking fuzzy numbers with a distance method using circumcenter of centroids and an Index of Modality, Advances in Fuzzy System, 10 (2011), 1155-1161. 